\title{
Veno-venous ECMO in patient with COVID-19 severe pneumonia
}

\author{
Marek Dabrowski ${ }^{1}$, Jacek Smereka ${ }^{2}$, Togay Evrin ${ }^{3}$, Burak Katipoglu ${ }^{3}$, Anna Drozd ${ }^{4}$, Lukasz \\ Szarpak $^{5}$
}

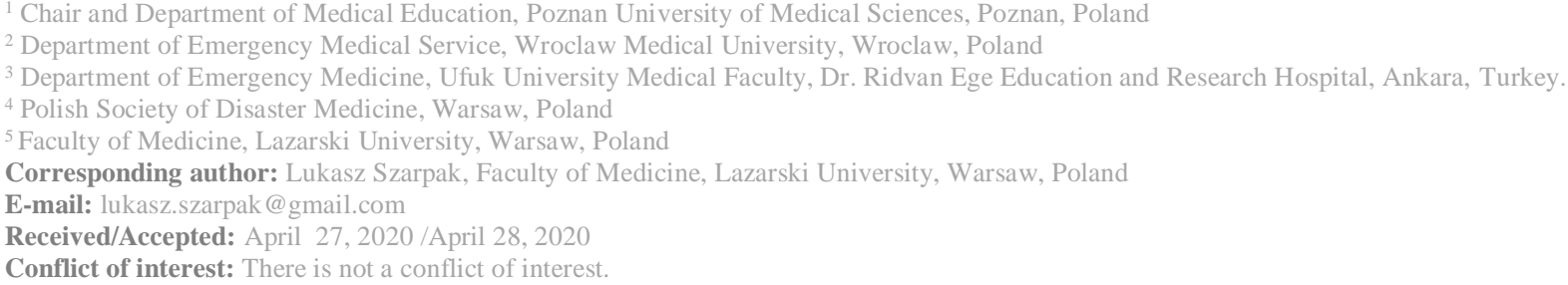

\section{To the Editor:}

since December 2019 we have been faced with a rapidly growing number of patients with COVID19, which is caused by the SARS-CoV-2 coronavirus ${ }^{1}$. According to the Centers for Disease Control and Prevention (CDC), COVID-19 is a respiratory illness. A person may have a dry cough, fever, muscle aches, and fatigue. Most common diagnosis for severe COVID-19 is severe pneumonia which may be life threatening ${ }^{2}$. In most cases, antiviral, antimicrobial, and steroidal treatment is applied in these patients. However, some cases are resistant to treatment ${ }^{3}$. Then the use of extracorporeal oxygenation membranes (ECMO) may be helpful.

ECMO is one of the methods of extracorporeal circulation and respiration support. The main goal of ECMO is to oxygenate blood and eliminate carbon dioxide outside the patient's body. The ECMO system consists of cannulas connecting extracorporeal circulation with the patient's vascular system, centrifugal pump, oxygenator, and drainage system (Figure 1). In the majority of cases, veno-venous ECMO is used, in which blood is collected and returned to the venous system. Most often in the system: right femoral vein as a drain of the venous system and right internal jugular vein as a return line to the patient ${ }^{4}$. This type only supports breathing when the blood is collected and returned to the venous system. In patients requiring not only respiratory support but also circulatory support, blood is collected from the venous system and returned to the arterial system, thus relieving the heart (venous arterial ECMO).

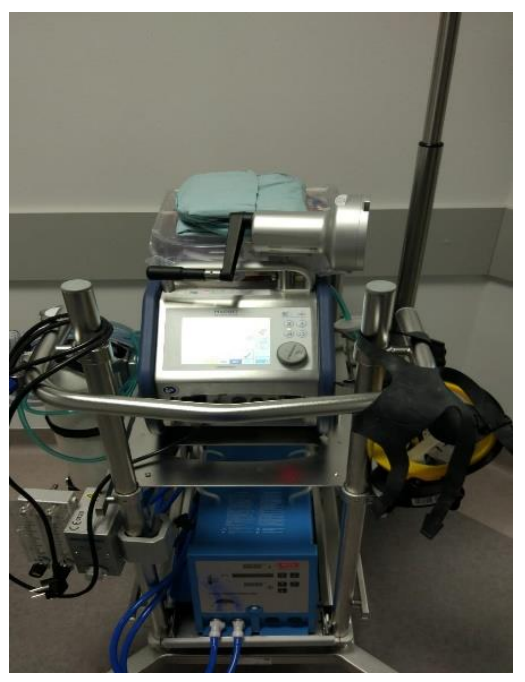

Figure 1. Extracorporeal membrane oxygenation device. 
It is worth remembering that the general indication for the use of ECMO is the occurrence of acute respiratory and/or circulatory failure resistant to conventional methods, which is based on a reversible and treatable disease process and the absence of absolute contraindications (i.e. irreversibility of the disease process underlying the respiratory and/or circulatory failure; contraindications for prolonged heparinisation; significant deterioration of the quality of life and functioning of the body after the completion of therapy, lack of consent of the patient or his legal guardians). ECMO should be considered for ARDS as rescue therapy when meeting one of the following criteria: (1) $\mathrm{PaO}_{2} / \mathrm{FiO}_{2}<100 \mathrm{mmHg}$; (2) $\mathrm{P}(\mathrm{A}-\mathrm{a}) \mathrm{O}_{2}>600 \mathrm{mmHg}$; (3) $\mathrm{pH}<7.2$ and plateau pressure $>30 \mathrm{cmH}_{2} \mathrm{O}$ with a respiratory rate more than 35 breaths per minute; (4) Age $<65$ years old; (5) Mechanical ventilation $<7 \mathrm{~d}$; (6) Absence of contraindications ${ }^{5}$.

According to the interim guidance formulated by the World Health Organization (WHO), ECMO should be considered as rescue therapy for COVID-19 with refractory hypoxemia despite lung-protective ventilation ${ }^{6}$. Also studies by Jacobs et al. indicate that ECMO may play a useful role in salvaging select critically ill patients with COVID-19 ${ }^{7}$.

During ECMO therapy, mechanical ventilation should not be forgotten. As indicated Burns et al. ${ }^{8}$ low-tidal-volume ventilation strategies improve survival. Moreover, positive airway pressure settings will also affect right and left ventricular load in both VV-ECMO as well as VA-ECMO ${ }^{9}$.

In summary, severe acute respiratory distress syndrome still has a high mortality rate, but ECMO may be able to improve the outcome. VV ECMO should be considered for patients with lifethreatening hypoxemia or hypercapnia refractory to conventional mechanical Moreover, mechanical ventilation settings are important in ECMO patients to minimize further lung damage and improve outcomes

Keywords: Acute respiratory distress syndrome; veno-venous ECMO; COVID-19; pneumonia; SARS-CoV-2

\section{REFERENCES}

1. Smereka J, Szarpak L, Filipiak KJ. Modern medicine in COVID-19 era. Disaster Emerg Med J 2020; 5(2); DOI: 10.5603/DEMJ.a2020.0012

2. Dzieciatkowski T, Szarpak L, Filipiak KJ, Jaguszewski M, Ladny JR, Smereka J. COVID-19 challenge for modern medicine. Cardiol J. 2020 Apr 14. doi: 10.5603/CJ.a2020.0055.

3. Lai CC, Shih TP, Ko WC, Tang HJ, Hsueh PR. Severe acute respiratory syndrome coronavirus 2 (SARS-CoV-2) and coronavirus disease-2019 (COVID-19): The epidemic and the challenges. Int J Antimicrob Agents. 2020 Mar;55(3):105924.

doi: 10.1016/j.ijantimicag.2020.105924

4. Smereka J, Puslecki M, Ruetzler K, et al. Extracorporeal membrane oxygenation in COVID-19. Cardiol J. 2020 Apr 14. doi: 10.5603/CJ.a2020.0053.

5. Pravda NS, Pravda MS, Kornowski R, Orvin K. Extracorporeal membrane oxygenation therapy in the COVID-19 pandemic. Future Cardiol. 2020 Apr 17. doi: 10.2217/fca-20200040.

6. WHO. Clinical management of severe acute respiratory infection when novel coronavirus $(\mathrm{nCoV})$ infection is suspected. 2020. . (accessed April 20, 2020) https://www.who.int/docs/defaultsource/coronaviruse/clinical-managementofnovel-cov.pdf.

7. Jacobs JP, Stammers AH, St Louis J. Extracorporeal Membrane Oxygenation in the Treatment of Severe Pulmonary and Cardiac Compromise in COVID-19: Experience with 32 patients. ASAIO J. 2020 Apr 17. doi: 10.1097/MAT.0000000000001185.

8. Burns KE, Adhikari NK, Slutsky AS, Guyatt GH, Villar J, Zhang H, Zhou Q, Cook DJ, Stewart TE, Meade MO. Pressure and volume-limited ventilation for the ventilatory management of patients with acute lung injury: a systematic review and meta-analysis. PLoS One. 2011;6:e14623. doi: 10.1371/journal.pone.0014623.

9. Luecke T, Pelosi P. Clinical review: Positive end-expiratory pressure and cardiac output. Crit Care. 2005;9:607-621. doi: 10.1186/cc3877. 